Acta Crystallographica Section E

Structure Reports

Online
ISSN 1600-5368

\section{Hydrogen-bonding patterns in trimethoprim tetrafluoroborate}

In the title compound [systematic name: 2,4-diamino-5-(3,4,5trimethoxybenzyl)pyrimidinium tetrafluoroborate], $\mathrm{C}_{14} \mathrm{H}_{19^{-}}$ $\mathrm{N}_{4} \mathrm{O}_{3}{ }^{+} \cdot \mathrm{BF}_{4}{ }^{-}$, the trimethoprim (TMP) molecule is protonated at one of the pyrimidine $\mathrm{N}$ atoms. The protonated $\mathrm{N}$ atom and 2-amine group of the TMP cation interact with the tetrafluoroborate anion through a pair of $\mathrm{N}-\mathrm{H} \cdots \mathrm{F}$ hydrogen bonds [graph set $R_{2}^{2}(8)$ ]. The inversion-related TMP cations are linked through a pair of $\mathrm{N}-\mathrm{H} \cdots \mathrm{N}$ hydrogen bonds. The 2amine group of one TMP cation and the 4-amine group of another cation are bridged by a methoxy $\mathrm{O}$ atom, via $\mathrm{N}-$ H. . O hydrogen bonds.

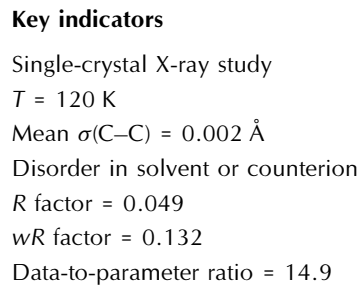

For details of how these key indicators were automatically derived from the article, see http://journals.iucr.org/e.
(C) 2005 International Union of Crystallography Printed in Great Britain - all rights reserved

\section{Comment}

Trimethoprim

[2,4-diamino-5-( $3^{\prime}, 4^{\prime}, 5^{\prime}$-trimethoxybenzyl)pyrimidine or TMP] is a well known antifolate drug. It is a potent inhibitor of bacterial dihydrofolate reductase (DHFR) but is less effective against human DHFR. The drug (TMP) in its N1-protonated form inhibits DHFR. The crystal structure of trimethoprim (Koetzle \& Williams, 1976) and its complexes, for example, trimethoprim monobenzoate benzoic acid (Bettinetti et al., 1985) and trimethoprim acetate (Bryan et al., 1987), have been reported in the literature. The present study has been undertaken to explore the hydrogen-bonding patterns involving the TMP cation in a variety of environments. The crystal structures of TMP sulfate trihydrate (Muthiah et al., 2001), TMP nitrate (Murugesan \& Muthiah, 1997) and TMP carboxylates (Stanley et al., 2005) have also been reported from our laboratory.<smiles>COc1cc(Cc2c[nH+]c(N)nc2N)cc(OC)c1OC</smiles>

(I)

The asymmetric unit of (I) contains a protonated trimethoprim (TMP) cation and a tetrafluoroborate anion (FLUB) (Fig. 1). The trimethoprim molecule is protonated at atom $\mathrm{N} 1$ of the pyrimidine moiety, which is evident from the increase in the internal angle at protonated $\mathrm{N} 1[\mathrm{C} 2-\mathrm{N} 1-\mathrm{C} 6$ $\left.=120.15(13)^{\circ}\right]$ compared with that at unprotonated atom N3 $\left[\mathrm{C} 2-\mathrm{N} 3-\mathrm{C} 4=118.36(14)^{\circ}\right]$. The dihedral angle between the pyrimidine and benzene planes is $84.27(7)^{\circ}$; the corresponding angle in trimethoprim perchlorate is $83.7(2)^{\circ}$ (Muthiah et al., 2002). The conformation of the trimethoprim cation is described by the two torsion angles $\mathrm{C} 4-\mathrm{C} 5-\mathrm{C} 7-$ $\mathrm{C} 8$ and $\mathrm{C} 5-\mathrm{C} 7-\mathrm{C} 8-\mathrm{C} 9$, which are $77.84(19)$ and
Received 3 October 2005 Accepted 8 November 2005 Online 16 November 2005 


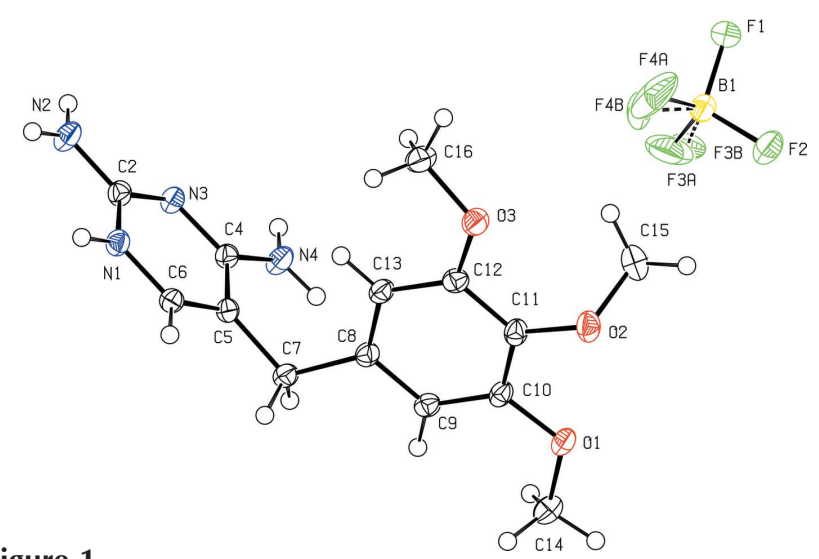

Figure 1

A view of (I), with the atom-labelling scheme and $50 \%$ probability displacement ellipsoids.

$-158.03(14)^{\circ}$, respectively. The distorted tetrahedral $\mathrm{BF}_{4}$ ion has typical $\mathrm{B}-\mathrm{F}$ distances.

Atoms $\mathrm{F} 2$ and $\mathrm{F} 1$ act as acceptors in $\mathrm{N}-\mathrm{H} \cdots \mathrm{F}$ interactions (Table 2) with the protonated pyrimidine $\mathrm{N} 1$ and 2-amine group of the TMP cation, leading to the formation of a forklike hydrogen-bonding pattern with graph-set notation $R_{2}^{2}(8)$ (Etter, 1990; Bernstein et al., 1995). The $R_{2}^{2}(8)$ motif is frequently observed in aminopyrimidine carboxylate salts (Lynch \& Jones, 2004). Here the tetrafluoroborate anion mimics the role of the carboxylate group. The TMP cations are paired centrosymmetrically through $\mathrm{N} 4-\mathrm{H} 4 A \cdots \mathrm{N} 3^{\mathrm{iii}}$ and $\mathrm{N} 3 \cdots \mathrm{H} 4 A^{\mathrm{iii}}-\mathrm{N} 4^{\mathrm{iii}}$ hydrogen bonds (symmetry codes are given in Table 2). The 2-amine group of one TMP cation and the 4-amine group of another cation (both of these cations being members of a base pair) are bridged by methoxy atom O1, using a pair of $\mathrm{N}-\mathrm{H} \cdots \mathrm{O}$ hydrogen bonds, leading to a complementary $D A D A(D=$ donor in hydrogen bonds, $A=$ acceptor in hydrogen bonds) array of quadruple hydrogen bonds (Fig. 2). This pattern is similar to that reported in TMP nitrate (Murugesan \& Muthiah, 1997), TMP trifluoroacetate (Francis et al., 2002), and TMP salicylate methanol solvate (Panneerselvam et al., 2002). The hydrogen-bonding parameters are listed in Table 2.

\section{Experimental}

Hot aqueous solutions of trimethoprim (145 mg; obtained as a gift sample from Shilpa Antibiotics Ltd) and tetrafluoroboric acid ( $220 \mathrm{mg}$ of $40 \%$ solution; Aldrich) were mixed in a 1:2 molar ratio. The resulting solution was warmed over a water bath for a few minutes and then kept at room temperature for crystallization. After a few days, block-shaped colourless crystals of (I) were obtained.

\footnotetext{
Crystal data

$\mathrm{C}_{14} \mathrm{H}_{19} \mathrm{~N}_{4} \mathrm{O}_{3}{ }^{+} \cdot \mathrm{BF}_{4}^{-}$

$M_{r}=378.14$

Triclinic, $P \overline{1}$

$a=9.4105(3) \AA$

$b=9.5397$ (2) $\AA$

$c=10.1276$ (4) $\AA$

$\alpha=88.564$ (2)

$\beta=73.253(2)^{\circ}$

$\gamma=72.304(2)^{\circ}$

$V=827.48(5) \AA^{3}$
}

\section{Data collection}

Bruker-Nonius KappaCCD areadetector diffractometer

$\varphi$ and $\omega$ scans

Absorption correction: none

18185 measured reflections

3811 independent reflections

Refinement

Refinement on $F^{2}$

$R\left[F^{2}>2 \sigma\left(F^{2}\right)\right]=0.049$

$w R\left(F^{2}\right)=0.132$

$S=1.15$

3811 reflections

255 parameters

$\mathrm{H}$-atom parameters constrained

$$
\begin{aligned}
& 3193 \text { reflections with } I>2 \sigma(I) \\
& R_{\text {int }}=0.041 \\
& \theta_{\max }=27.6^{\circ} \\
& h=-12 \rightarrow 12 \\
& k=-12 \rightarrow 12 \\
& l=-13 \rightarrow 13
\end{aligned}
$$

Table 1

Selected geometric parameters $\left(\AA \stackrel{\circ}{\circ}^{\circ}\right)$.

\begin{tabular}{llll}
\hline $\mathrm{O} 1-\mathrm{C} 10$ & $1.377(2)$ & $\mathrm{N} 1-\mathrm{C} 2$ & $1.356(2)$ \\
$\mathrm{O} 1-\mathrm{C} 14$ & $1.443(2)$ & $\mathrm{N} 1-\mathrm{C} 6$ & $1.364(2)$ \\
$\mathrm{O} 2-\mathrm{C} 11$ & $1.3802(19)$ & $\mathrm{N} 2-\mathrm{C} 2$ & $1.330(2)$ \\
$\mathrm{O} 2-\mathrm{C} 15$ & $1.435(2)$ & $\mathrm{N} 3-\mathrm{C} 2$ & $1.331(2)$ \\
$\mathrm{O} 3-\mathrm{C} 16$ & $1.435(2)$ & $\mathrm{N} 3-\mathrm{C} 4$ & $1.347(2)$ \\
$\mathrm{O} 3-\mathrm{C} 12$ & $1.368(2)$ & $\mathrm{N} 4-\mathrm{C} 4$ & $1.322(2)$ \\
& & & \\
$\mathrm{C} 10-\mathrm{O} 1-\mathrm{C} 14$ & $116.47(12)$ & $\mathrm{N} 4-\mathrm{C} 4-\mathrm{C} 5$ & $120.69(15)$ \\
$\mathrm{C} 11-\mathrm{O} 2-\mathrm{C} 15$ & $114.69(13)$ & $\mathrm{N} 3-\mathrm{C} 4-\mathrm{N} 4$ & $117.24(15)$ \\
$\mathrm{C} 12-\mathrm{O} 3-\mathrm{C} 16$ & $116.53(12)$ & $\mathrm{N} 1-\mathrm{C} 6-\mathrm{C} 5$ & $121.27(15)$ \\
$\mathrm{C} 2-\mathrm{N} 1-\mathrm{C} 6$ & $120.15(13)$ & $\mathrm{O} 1-\mathrm{C} 10-\mathrm{C} 9$ & $123.88(15)$ \\
$\mathrm{C} 2-\mathrm{N} 3-\mathrm{C} 4$ & $118.36(14)$ & $\mathrm{O} 1-\mathrm{C} 10-\mathrm{C} 11$ & $115.49(14)$ \\
$\mathrm{N} 2-\mathrm{C} 2-\mathrm{N} 3$ & $119.35(15)$ & $\mathrm{O} 2-\mathrm{C} 11-\mathrm{C} 12$ & $121.87(15)$ \\
$\mathrm{N} 1-\mathrm{C} 2-\mathrm{N} 2$ & $118.59(14)$ & $\mathrm{O} 2-\mathrm{C} 11-\mathrm{C} 10$ & $118.96(15)$ \\
$\mathrm{N} 1-\mathrm{C} 2-\mathrm{N} 3$ & $122.06(15)$ & $\mathrm{O} 3-\mathrm{C} 12-\mathrm{C} 11$ & $115.08(14)$ \\
$\mathrm{N} 3-\mathrm{C} 4-\mathrm{C} 5$ & $122.06(14)$ & $\mathrm{O} 3-\mathrm{C} 12-\mathrm{C} 13$ & $124.55(15)$ \\
\hline
\end{tabular}

Table 2

Hydrogen-bond geometry $\left(\AA,{ }^{\circ}\right)$.

\begin{tabular}{lllll}
\hline$D-\mathrm{H} \cdots A$ & $D-\mathrm{H}$ & $\mathrm{H} \cdots A$ & $D \cdots A$ & $D-\mathrm{H} \cdots A$ \\
\hline $\mathrm{N} 1-\mathrm{H} 1 \cdots \mathrm{F} 2^{\mathrm{i}}$ & 0.88 & 1.97 & $2.8445(17)$ & 177 \\
$\mathrm{~N} 2-\mathrm{H} 2 A \cdots \mathrm{O} 1^{\mathrm{ii}}$ & 0.88 & 2.10 & $2.9345(18)$ & 158 \\
$\mathrm{~N} 2-\mathrm{H} 2 B \cdots 1^{\mathrm{i}}$ & 0.88 & 2.01 & $2.8851(17)$ & 174 \\
$\mathrm{~N} 4-\mathrm{H} 4 A \cdots \mathrm{N} 3^{\text {iii }}$ & 0.88 & 2.29 & $3.121(2)$ & 158 \\
$\mathrm{~N} 4-\mathrm{H} 4 B \cdots \mathrm{O} 1^{\text {iv }}$ & 0.88 & 2.36 & $2.9210(18)$ & 122 \\
$\mathrm{C} 6-\mathrm{H} 6 \cdots \mathrm{O} 2^{\mathrm{v}}$ & 0.95 & 2.50 & $3.171(2)$ & 128 \\
$\mathrm{C} 9-\mathrm{H} 9 \cdots \mathrm{F} 3 A^{\text {iv }}$ & 0.95 & 2.38 & $2.995(9)$ & 122 \\
$\mathrm{C} 14-\mathrm{H} 14 B \cdots \mathrm{F} 4 A^{\mathrm{vi}}$ & 0.98 & 2.50 & $3.414(10)$ & 155 \\
$\mathrm{C} 15-\mathrm{H} 15 C \cdots \mathrm{O} 3$ & 0.98 & 2.46 & $3.022(2)$ & 116
\end{tabular}

Symmetry codes: (i) $x, y+1, z-1$; (ii) $x+1, y, z-1$; (iii) $-x+1,-y+1,-z$; (iv) $-x,-y+1,-z+1 ;$ (v) $-x,-y+2,-z+1$; (vi) $x-1, y, z$.

All $\mathrm{H}$ atoms were placed in idealized locations and were refined using a riding model, with $\mathrm{C}-\mathrm{H}=0.95-0.99 \AA, \mathrm{N}-\mathrm{H}=0.88 \AA$ and $U_{\text {iso }}(\mathrm{H})=1.2 U_{\text {eq }}(\mathrm{C}, \mathrm{N})$. Two of the $\mathrm{F}$ atoms in the $\mathrm{BF}_{4}$ group are disordered over two positions, and the occupancy factors for the disordered positions $\mathrm{F} 3 A / \mathrm{F} 3 B$ and $\mathrm{F} 4 A / \mathrm{F} 4 B$ were refined to 0.60 (2)/ 0.40 (2). Similarity restraints were applied to distances involving disordered atoms.

Data collection: COLLECT (Hooft, 1998); cell refinement: DENZO (Otwinowski \& Minor, 1997) and COLLECT; data reduction: DENZO and COLLECT; program(s) used to solve structure: SHELXS97 (Sheldrick, 1997); program(s) used to refine structure: 


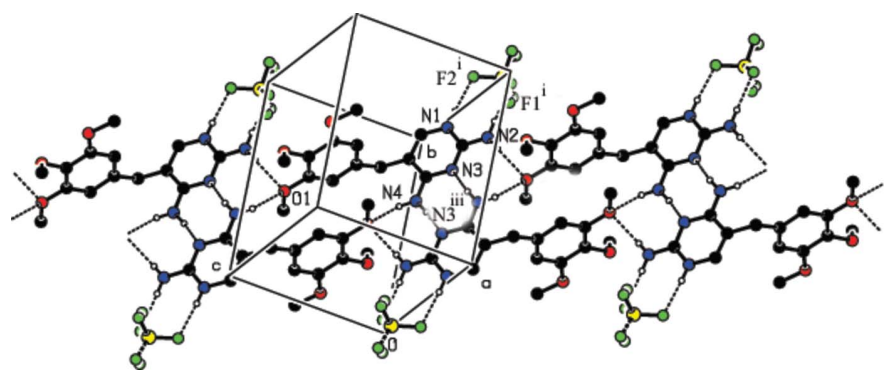

Figure 2

The hydrogen-bonding (dashed lines) patterns of (I). [Symmetry codes: (i) $x, 1+y, z-1$; (iii) $1-x, 1-y,-z$.]

SHELXL97 (Sheldrick, 1997); molecular graphics: PLATON (Spek, 2003); software used to prepare material for publication: PLATON.

$\mathrm{MH}$ thanks the Council of Scientific and Industrial Research (CSIR), India, for the award of a Senior Research Fellowship (SRF) [reference No. 9/475(123)/2004-EMR-I]. DL thanks the EPSRC National Crystallography Service (Southampton, England) for X-ray data collection.

\section{References}

Bernstein, J., Davis, R. E., Shimoni, L. \& Chang, N.-L. (1995). Angew. Chem. Int. Ed. Engl. 34, 1555-1573.

Bettinetti, G. P., Giordano, F., La Manna, A., Giuseppetti, G. \& Tadini, C. (1985). Acta Cryst. C41, 1249-1253.

Bryan, R. F., Haltiwanger, R. C. \& Woods, M. K. (1987). Acta Cryst. C43, $2412-$ 2415.

Etter, M. C. (1990). Acc. Chem. Res. 23, 120-126.

Francis, S., Muthiah, P. T., Bocelli, G. \& Righi, L. (2002). Acta Cryst. E58, o717-0719.

Hooft, R. (1998). COLLECT. Nonius BV, Delft, The Netherlands.

Koetzle, T. F. \& Williams, G. J. B. (1976). J. Am. Chem. Soc. 98, 2074-2078.

Lynch, D. E. \& Jones, G. D. (2004). Acta Cryst. B60, 748-754.

Murugesan, S. \& Muthiah, P. T. (1997). Acta Cryst. C53, 763-764.

Muthiah, P. T., Umadevi, B., Stanley, N., Bocelli, G. \& Cantoni, A. (2002). Acta Cryst. E58, o59-o61.

Muthiah, P. T., Umadevi, B., Stanley, N., Shui, X. \& Eggleston, D. S. (2001). Acta Cryst. E57, o1179-o1182.

Otwinowski, Z. \& Minor, W. (1997). Methods in Enzymology. Vol. 276, Macromolecular Crystallography, Part A, edited by C. W. Carter Jr \& R. M. Sweet, pp. 307-326. New York: Academic Press.

Panneerselvam, P., Stanley, N. \& Muthiah, P. T. (2002). Acta Cryst. E58, o180o182.

Sheldrick, G. M. (1997). SHELXS97 and SHELXL97. University of Göttingen, Germany.

Spek, A. L. (2003). J. Appl. Cryst. 36, 7-13.

Stanley, N., Muthiah, P. T., Geib, S. J., Luger, P., Weber, M. \& Messerschmidt, M. (2005). Tetrahedron, 61, 7201-7210. 


\section{supporting information}

Acta Cryst. (2005). E61, o4107-o4109 [https://doi.org/10.1107/S1600536805036615]

Hydrogen-bonding patterns in trimethoprim tetrafluoroborate

Madhukar Hemamalini, Packianathan Thomas Muthiah and Daniel E. Lynch

2,4-diamino-5-(3,4,5-trimethoxybenzyl)pyrimidinium fluoroborate

Crystal data

$\mathrm{C}_{14} \mathrm{H}_{19} \mathrm{~N}_{4} \mathrm{O}_{3}{ }^{+} \cdot \mathrm{BF}_{4}^{-}$

$M_{r}=378.14$

Triclinic, $P \overline{1}$

Hall symbol: -P 1

$a=9.4105(3) \AA$

$b=9.5397(2) \AA$

$c=10.1276(4) \AA$

$\alpha=88.564(2)^{\circ}$

$\beta=73.253(2)^{\circ}$

$\gamma=72.304(2)^{\circ}$

$V=827.48(5) \AA^{3}$

\section{Data collection}

Bruker-Nonius KappaCCD area-detector diffractometer

Radiation source: Bruker-Nonius FR591 rotating anode

Graphite monochromator

$\varphi$ and $\omega$ scans

18185 measured reflections

\section{Refinement}

Refinement on $F^{2}$

Least-squares matrix: full

$R\left[F^{2}>2 \sigma\left(F^{2}\right)\right]=0.049$

$w R\left(F^{2}\right)=0.132$

$S=1.15$

3811 reflections

255 parameters

6 restraints

Primary atom site location: structure-invariant direct methods

Secondary atom site location: difference Fourier map

\section{Special details}

Geometry. Bond distances, angles etc. have been calculated using the rounded fractional coordinates. All e.s.d.'s are estimated from the variances of the (full) variance-covariance matrix. The cell e.s.d.'s are taken into account in the estimation of distances, angles and torsion angles
$Z=2$

$F(000)=392$

$D_{\mathrm{x}}=1.518 \mathrm{Mg} \mathrm{m}^{-3}$

Mo $K \alpha$ radiation, $\lambda=0.71073 \AA$

Cell parameters from 3811 reflections

$\theta=3.0-27.6^{\circ}$

$\mu=0.14 \mathrm{~mm}^{-1}$

$T=120 \mathrm{~K}$

Block, colourless

$0.18 \times 0.16 \times 0.09 \mathrm{~mm}$

3811 independent reflections

3193 reflections with $I>2 \sigma(I)$

$R_{\text {int }}=0.041$

$\theta_{\max }=27.6^{\circ}, \theta_{\min }=3.0^{\circ}$

$h=-12 \rightarrow 12$

$k=-12 \rightarrow 12$

$l=-13 \rightarrow 13$

Hydrogen site location: inferred from neighbouring sites

$\mathrm{H}$-atom parameters constrained

$w=1 /\left[\sigma^{2}\left(F_{\mathrm{o}}^{2}\right)+(0.0644 P)^{2}+0.2497 P\right]$

where $P=\left(F_{\mathrm{o}}{ }^{2}+2 F_{\mathrm{c}}{ }^{2}\right) / 3$

$(\Delta / \sigma)_{\max }=0.001$

$\Delta \rho_{\max }=0.61 \mathrm{e} \AA^{-3}$

$\Delta \rho_{\min }=-0.52$ e $\AA^{-3}$

Extinction correction: SHELXL97, $\mathrm{FC}^{*}=\mathrm{KFC}\left[1+0.001 \mathrm{XFC}^{2} \Lambda^{3} / \mathrm{SIN}(2 \Theta)\right]^{-1 / 4}$

Extinction coefficient: $0.133(9)$ 
Refinement. Refinement on $F^{2}$ for ALL reflections except those flagged by the user for potential systematic errors. Weighted $R$-factors $w R$ and all goodnesses of fit $S$ are based on $F^{2}$, conventional $R$-factors $R$ are based on $F$, with $F$ set to zero for negative $F^{2}$. The observed criterion of $F^{2}>\sigma\left(F^{2}\right)$ is used only for calculating - $R$-factor-obs etc. and is not relevant to the choice of reflections for refinement. $R$-factors based on $F^{2}$ are statistically about twice as large as those based on $F$, and $R$-factors based on ALL data will be even larger.

Fractional atomic coordinates and isotropic or equivalent isotropic displacement parameters $\left(\AA^{2}\right)$

\begin{tabular}{|c|c|c|c|c|c|}
\hline & $x$ & $y$ & $z$ & $U_{\text {iso }} * / U_{\text {eq }}$ & Occ. $(<1)$ \\
\hline $\mathrm{O} 1$ & $-0.22859(13)$ & $0.61645(12)$ & $0.66705(11)$ & 0.0221 & \\
\hline $\mathrm{O} 2$ & $-0.06715(14)$ & $0.72735(13)$ & $0.78897(11)$ & $0.0248(3)$ & \\
\hline $\mathrm{O} 3$ & $0.15105(13)$ & $0.84755(12)$ & $0.64102(11)$ & $0.0235(3)$ & \\
\hline N1 & $0.28278(15)$ & $0.96385(14)$ & $-0.00878(13)$ & $0.0199(4)$ & \\
\hline N2 & $0.53520(16)$ & $0.86442(15)$ & $-0.15031(14)$ & $0.0246(4)$ & \\
\hline N3 & $0.40722(15)$ & $0.70767(14)$ & $-0.03325(13)$ & $0.0195(4)$ & \\
\hline N4 & $0.28298(16)$ & $0.55383(14)$ & $0.09004(15)$ & $0.0238(4)$ & \\
\hline $\mathrm{C} 2$ & $0.40794(18)$ & $0.84404(17)$ & $-0.06383(15)$ & $0.0190(4)$ & \\
\hline $\mathrm{C} 4$ & $0.27950(18)$ & $0.68998(17)$ & $0.05895(15)$ & $0.0186(4)$ & \\
\hline $\mathrm{C} 5$ & $0.14285(18)$ & $0.81297(17)$ & $0.12055(15)$ & $0.0182(4)$ & \\
\hline C6 & $0.15165(18)$ & $0.94728(17)$ & $0.08297(16)$ & $0.0192(4)$ & \\
\hline $\mathrm{C} 7$ & $-0.00296(17)$ & $0.79327(17)$ & $0.21807(16)$ & $0.0199(4)$ & \\
\hline $\mathrm{C} 8$ & $-0.00211(17)$ & $0.76276(16)$ & $0.36594(15)$ & $0.0185(4)$ & \\
\hline C9 & -0.10785 (17) & 0.69467 (16) & $0.44282(16)$ & $0.0190(4)$ & \\
\hline $\mathrm{C} 10$ & $-0.12541(17)$ & $0.68018(16)$ & $0.58268(16)$ & $0.0188(4)$ & \\
\hline C11 & $-0.03807(18)$ & $0.73361(16)$ & $0.64772(16)$ & $0.0196(4)$ & \\
\hline $\mathrm{C} 12$ & $0.07051(18)$ & $0.79892(16)$ & $0.56898(16)$ & $0.0196(4)$ & \\
\hline C13 & $0.08929(18)$ & $0.81245(17)$ & $0.42804(16)$ & $0.0194(4)$ & \\
\hline $\mathrm{C} 14$ & $-0.3298(2)$ & $0.57224(19)$ & $0.60493(18)$ & $0.0266(5)$ & \\
\hline $\mathrm{C} 15$ & $0.0542(2)$ & 0.62255 (19) & $0.83157(18)$ & $0.0298(5)$ & \\
\hline $\mathrm{C} 16$ & $0.28441(19)$ & $0.8868(2)$ & $0.56058(18)$ & $0.0266(5)$ & \\
\hline $\mathrm{F} 1$ & $0.56011(11)$ & $0.15397(11)$ & $0.78546(10)$ & 0.0272 & \\
\hline $\mathrm{F} 2$ & $0.30228(12)$ & $0.24550(11)$ & $0.90592(11)$ & $0.0322(3)$ & \\
\hline F3A & $0.3745(7)$ & $0.1814(13)$ & $0.6750(7)$ & $0.083(2)$ & $0.60(2)$ \\
\hline F4A & $0.4213(9)$ & $0.3796(4)$ & $0.7583(15)$ & $0.083(2)$ & $0.60(2)$ \\
\hline B1 & $0.4133(2)$ & 0.23827 (19) & $0.7771(2)$ & $0.0276(6)$ & \\
\hline F3B & $0.3813(8)$ & $0.1471(6)$ & $0.6919(6)$ & $0.0282(16)$ & $0.40(2)$ \\
\hline F4B & $0.4046(11)$ & $0.3723(5)$ & $0.7221(6)$ & $0.0322(16)$ & $0.40(2)$ \\
\hline $\mathrm{H} 1$ & 0.28620 & 1.05250 & -0.03210 & $0.0240 *$ & \\
\hline $\mathrm{H} 2 \mathrm{~A}$ & 0.61790 & 0.78820 & -0.18670 & $0.0300^{*}$ & \\
\hline $\mathrm{H} 2 \mathrm{~B}$ & 0.53670 & 0.95420 & -0.17110 & $0.0300^{*}$ & \\
\hline $\mathrm{H} 4 \mathrm{~A}$ & 0.36650 & 0.47940 & 0.05090 & $0.0290 *$ & \\
\hline $\mathrm{H} 4 \mathrm{~B}$ & 0.20180 & 0.53760 & 0.14980 & $0.0290 *$ & \\
\hline H6 & 0.06490 & 1.03210 & 0.12110 & $0.0230 *$ & \\
\hline H7A & -0.02500 & 0.71090 & 0.17900 & $0.0240 *$ & \\
\hline H7B & -0.09060 & 0.88360 & 0.22080 & $0.0240 *$ & \\
\hline H9 & -0.16780 & 0.65820 & 0.39950 & $0.0230 *$ & \\
\hline H13 & 0.16410 & 0.85550 & 0.37460 & $0.0230 *$ & \\
\hline H14A & -0.39000 & 0.65770 & 0.56720 & $0.0320 *$ & \\
\hline
\end{tabular}




\begin{tabular}{lllll} 
H14B & -0.40140 & 0.53330 & 0.67510 & $0.0320^{*}$ \\
H14C & -0.26680 & 0.49570 & 0.53040 & $0.0320^{*}$ \\
H15A & 0.07690 & 0.52510 & 0.78690 & $0.0360^{*}$ \\
H15B & 0.02010 & 0.61800 & 0.93220 & $0.0360^{*}$ \\
H15C & 0.14860 & 0.65310 & 0.80480 & $0.0360^{*}$ \\
H16A & 0.35430 & 0.80460 & 0.49390 & $0.0320^{*}$ \\
H16B & 0.34000 & 0.90840 & 0.62190 & $0.0320^{*}$ \\
H16C & 0.25000 & 0.97420 & 0.51100 & $0.0320^{*}$ \\
\hline
\end{tabular}

Atomic displacement parameters $\left(\AA^{2}\right)$

\begin{tabular}{|c|c|c|c|c|c|c|}
\hline & $U^{11}$ & $U^{22}$ & $U^{33}$ & $U^{12}$ & $U^{13}$ & $U^{23}$ \\
\hline $\mathrm{O} 1$ & $0.0211(6)$ & $0.0236(6)$ & $0.0208(6)$ & $-0.0100(5)$ & $-0.0018(5)$ & $0.0043(4)$ \\
\hline $\mathrm{O} 2$ & $0.0277(6)$ & $0.0273(6)$ & $0.0159(6)$ & $-0.0064(5)$ & $-0.0036(5)$ & $0.0030(4)$ \\
\hline $\mathrm{O} 3$ & $0.0241(6)$ & $0.0297(6)$ & $0.0193(6)$ & $-0.0123(5)$ & $-0.0059(5)$ & 0.0008 (4) \\
\hline N1 & $0.0206(7)$ & $0.0182(6)$ & $0.0204(7)$ & $-0.0069(5)$ & $-0.0045(5)$ & $0.0037(5)$ \\
\hline $\mathrm{N} 2$ & $0.0212(7)$ & $0.0191(7)$ & $0.0281(7)$ & $-0.0068(5)$ & $0.0014(6)$ & $0.0036(5)$ \\
\hline N3 & $0.0195(7)$ & $0.0190(6)$ & $0.0183(6)$ & $-0.0064(5)$ & $-0.0026(5)$ & $0.0019(5)$ \\
\hline N4 & $0.0194(7)$ & $0.0195(7)$ & $0.0271(7)$ & $-0.0058(5)$ & $0.0008(6)$ & $0.0036(5)$ \\
\hline $\mathrm{C} 2$ & $0.0205(8)$ & $0.0214(8)$ & $0.0162(7)$ & $-0.0079(6)$ & $-0.0054(6)$ & $0.0016(6)$ \\
\hline $\mathrm{C} 4$ & $0.0189(7)$ & $0.0219(8)$ & $0.0163(7)$ & $-0.0081(6)$ & $-0.0054(6)$ & $0.0024(6)$ \\
\hline $\mathrm{C} 5$ & $0.0178(7)$ & $0.0233(8)$ & $0.0142(7)$ & $-0.0071(6)$ & $-0.0048(6)$ & $0.0016(6)$ \\
\hline C6 & $0.0173(7)$ & $0.0224(8)$ & $0.0168(7)$ & $-0.0051(6)$ & $-0.0043(6)$ & $0.0008(6)$ \\
\hline $\mathrm{C} 7$ & $0.0162(7)$ & $0.0239(8)$ & $0.0185(7)$ & $-0.0066(6)$ & $-0.0032(6)$ & $0.0010(6)$ \\
\hline $\mathrm{C} 8$ & $0.0158(7)$ & $0.0184(7)$ & $0.0174(7)$ & $-0.0027(6)$ & $-0.0017(6)$ & $0.0007(5)$ \\
\hline C9 & $0.0161(7)$ & $0.0175(7)$ & $0.0218(8)$ & $-0.0046(6)$ & $-0.0039(6)$ & $0.0005(6)$ \\
\hline $\mathrm{C} 10$ & $0.0166(7)$ & $0.0152(7)$ & $0.0202(8)$ & $-0.0035(6)$ & $-0.0003(6)$ & $0.0023(6)$ \\
\hline $\mathrm{C} 11$ & $0.0196(7)$ & $0.0187(7)$ & $0.0162(7)$ & $-0.0032(6)$ & $-0.0016(6)$ & $0.0011(5)$ \\
\hline C12 & $0.0170(7)$ & $0.0188(7)$ & $0.0208(8)$ & $-0.0038(6)$ & $-0.0042(6)$ & $0.0000(6)$ \\
\hline C13 & $0.0162(7)$ & $0.0210(7)$ & $0.0189(7)$ & $-0.0058(6)$ & $-0.0018(6)$ & $0.0012(6)$ \\
\hline C14 & $0.0245(8)$ & $0.0283(9)$ & $0.0282(9)$ & $-0.0136(7)$ & $-0.0040(7)$ & $0.0029(7)$ \\
\hline $\mathrm{C} 15$ & $0.0392(10)$ & $0.0273(9)$ & $0.0239(9)$ & $-0.0084(7)$ & $-0.0132(8)$ & $0.0054(7)$ \\
\hline $\mathrm{C} 16$ & $0.0216(8)$ & $0.0341(9)$ & $0.0254(8)$ & $-0.0124(7)$ & $-0.0045(7)$ & $-0.0010(7)$ \\
\hline F1 & $0.0221(5)$ & $0.0325(5)$ & $0.0288(5)$ & $-0.0086(4)$ & $-0.0101(4)$ & 0.0049 (4) \\
\hline $\mathrm{F} 2$ & $0.0281(6)$ & $0.0300(5)$ & $0.0319(6)$ & $-0.0094(4)$ & 0.0015 (4) & 0.0051 (4) \\
\hline F3A & $0.0294(18)$ & $0.177(6)$ & $0.0344(18)$ & $-0.012(3)$ & $-0.0181(14)$ & $0.006(3)$ \\
\hline F4A & $0.036(2)$ & $0.041(2)$ & $0.137(6)$ & $-0.0033(16)$ & $0.015(4)$ & $0.052(3)$ \\
\hline B1 & $0.0211(9)$ & $0.0302(10)$ & $0.0292(10)$ & $-0.0077(8)$ & $-0.0052(8)$ & $0.0113(8)$ \\
\hline F3B & $0.027(2)$ & $0.028(3)$ & $0.037(3)$ & $-0.0106(13)$ & $-0.0177(18)$ & $-0.0065(15)$ \\
\hline F4B & $0.038(3)$ & $0.025(2)$ & $0.027(3)$ & $-0.0061(16)$ & $-0.0042(15)$ & $0.0111(15)$ \\
\hline
\end{tabular}

Geometric parameters $\left(\AA,{ }^{\circ}\right)$

\begin{tabular}{llll}
\hline F1-B1 & $1.397(2)$ & $\mathrm{C} 5-\mathrm{C} 7$ & $1.503(2)$ \\
F2-B1 & $1.405(2)$ & $\mathrm{C} 5-\mathrm{C} 6$ & $1.347(2)$ \\
F3A-B1 & $1.365(8)$ & $\mathrm{C} 7-\mathrm{C} 8$ & $1.520(2)$ \\
F3B-B1 & $1.392(7)$ & $\mathrm{C} 8-\mathrm{C} 9$ & $1.393(2)$ \\
F4A-B1 & $1.379(5)$ & $\mathrm{C} 8-\mathrm{C} 13$ & $1.391(2)$
\end{tabular}




\begin{tabular}{|c|c|c|c|}
\hline $\mathrm{F} 4 \mathrm{~B}-\mathrm{B} 1$ & $1.371(5)$ & $\mathrm{C} 9-\mathrm{C} 10$ & $1.386(2)$ \\
\hline $\mathrm{O} 1-\mathrm{C} 10$ & $1.377(2)$ & $\mathrm{C} 10-\mathrm{C} 11$ & $1.397(2)$ \\
\hline $\mathrm{O} 1-\mathrm{C} 14$ & $1.443(2)$ & $\mathrm{C} 11-\mathrm{C} 12$ & $1.400(2)$ \\
\hline $\mathrm{O} 2-\mathrm{C} 11$ & $1.3802(19)$ & $\mathrm{C} 12-\mathrm{C} 13$ & $1.394(2)$ \\
\hline $\mathrm{O} 2-\mathrm{C} 15$ & $1.435(2)$ & $\mathrm{C} 6-\mathrm{H} 6$ & 0.95 \\
\hline $\mathrm{O} 3-\mathrm{C} 16$ & $1.435(2)$ & $\mathrm{C} 7-\mathrm{H} 7 \mathrm{~A}$ & 0.99 \\
\hline $\mathrm{O} 3-\mathrm{C} 12$ & $1.368(2)$ & $\mathrm{C} 7-\mathrm{H} 7 \mathrm{~B}$ & 0.99 \\
\hline $\mathrm{N} 1-\mathrm{C} 2$ & $1.356(2)$ & C9-H9 & 0.95 \\
\hline $\mathrm{N} 1-\mathrm{C} 6$ & 1.364 (2) & $\mathrm{C} 13-\mathrm{H} 13$ & 0.95 \\
\hline $\mathrm{N} 2-\mathrm{C} 2$ & $1.330(2)$ & $\mathrm{C} 14-\mathrm{H} 14 \mathrm{~B}$ & 0.98 \\
\hline $\mathrm{N} 3-\mathrm{C} 2$ & $1.331(2)$ & $\mathrm{C} 14-\mathrm{H} 14 \mathrm{~A}$ & 0.98 \\
\hline $\mathrm{N} 3-\mathrm{C} 4$ & $1.347(2)$ & $\mathrm{C} 14-\mathrm{H} 14 \mathrm{C}$ & 0.98 \\
\hline $\mathrm{N} 4-\mathrm{C} 4$ & $1.322(2)$ & $\mathrm{C} 15-\mathrm{H} 15 \mathrm{~B}$ & 0.98 \\
\hline $\mathrm{N} 1-\mathrm{H} 1$ & 0.88 & $\mathrm{C} 15-\mathrm{H} 15 \mathrm{~A}$ & 0.98 \\
\hline $\mathrm{N} 2-\mathrm{H} 2 \mathrm{~B}$ & 0.88 & $\mathrm{C} 15-\mathrm{H} 15 \mathrm{C}$ & 0.98 \\
\hline $\mathrm{N} 2-\mathrm{H} 2 \mathrm{~A}$ & 0.88 & $\mathrm{C} 16-\mathrm{H} 16 \mathrm{C}$ & 0.98 \\
\hline $\mathrm{N} 4-\mathrm{H} 4 \mathrm{~A}$ & 0.88 & $\mathrm{C} 16-\mathrm{H} 16 \mathrm{~A}$ & 0.98 \\
\hline N4-H4B & 0.88 & $\mathrm{C} 16-\mathrm{H} 16 \mathrm{~B}$ & 0.98 \\
\hline $\mathrm{C} 4-\mathrm{C} 5$ & $1.441(2)$ & & \\
\hline $\mathrm{F} 1 \cdots \mathrm{C} 16^{\mathrm{i}}$ & $3.368(2)$ & $\mathrm{C} 9 \cdots \mathrm{H} 15 \mathrm{~A}^{\mathrm{iii}}$ & 3.0620 \\
\hline $\mathrm{F} 1 \cdots \mathrm{C} 2^{\mathrm{i}}$ & $2.9212(18)$ & $\mathrm{C} 9 \cdots \mathrm{H} 14 \mathrm{C}$ & 2.7394 \\
\hline $\mathrm{F} 1 \cdots \mathrm{N} 1^{\mathrm{i}}$ & $3.0584(17)$ & $\mathrm{C} 9 \cdots \mathrm{H} 16 \mathrm{C}^{\mathrm{vii}}$ & 3.0218 \\
\hline $\mathrm{F} 1 \cdots \mathrm{N} 3^{\mathrm{i}}$ & $2.9986(17)$ & $\mathrm{C} 10 \cdots \mathrm{H} 2 \mathrm{~A}^{\mathrm{vi}}$ & 2.7763 \\
\hline $\mathrm{F} 1 \cdots \mathrm{N} 2^{\mathrm{ii}}$ & $2.8851(17)$ & $\mathrm{C} 11 \cdots \mathrm{H} 2 \mathrm{~A}^{\mathrm{vi}}$ & 3.0749 \\
\hline $\mathrm{F} 1 \cdots \mathrm{C} 4^{\mathrm{i}}$ & $3.136(2)$ & $\mathrm{C} 12 \cdots \mathrm{H} 14 \mathrm{C}^{\mathrm{iii}}$ & 2.8703 \\
\hline $\mathrm{F} 1 \cdots \mathrm{C} 5^{\mathrm{i}}$ & $3.302(2)$ & $\mathrm{C} 12 \cdots \mathrm{H} 15 \mathrm{C}$ & 2.8987 \\
\hline $\mathrm{F} 1 \cdots \mathrm{C} 6^{\mathrm{i}}$ & $3.239(2)$ & $\mathrm{C} 13 \cdots \mathrm{H} 16 \mathrm{C}$ & 2.7550 \\
\hline $\mathrm{F} 2 \cdots \mathrm{N} 1^{\mathrm{ii}}$ & $2.8445(17)$ & $\mathrm{C} 13 \cdots \mathrm{H} 16 \mathrm{~A}$ & 2.7409 \\
\hline $\mathrm{F} 3 \mathrm{~A} \cdots \mathrm{C} 16^{\mathrm{i}}$ & $3.288(8)$ & $\mathrm{C} 13 \cdots \mathrm{H} 14 \mathrm{C}^{\mathrm{iii}}$ & 2.9945 \\
\hline $\mathrm{F} 3 \mathrm{~A} \cdots \mathrm{C} 9^{\mathrm{iii}}$ & $2.995(9)$ & $\mathrm{C} 14 \cdots \mathrm{H} 2 \mathrm{~A}^{\mathrm{vi}}$ & 2.8183 \\
\hline $\mathrm{F} 3 \mathrm{~A} \cdots \mathrm{C} 7^{\mathrm{iii}}$ & $3.282(8)$ & $\mathrm{C} 14 \cdots \mathrm{H}_{4} \mathrm{~B}^{\mathrm{iii}}$ & 3.0896 \\
\hline $\mathrm{F} 3 \mathrm{~B} \cdots \mathrm{C} 16^{\mathrm{iv}}$ & $3.318(7)$ & $\mathrm{C} 14 \cdots \mathrm{H} 9$ & 2.4843 \\
\hline $\mathrm{F} 3 \mathrm{~B} \cdots \mathrm{C} 7^{\mathrm{iii}}$ & $3.279(8)$ & $\mathrm{C} 16 \cdots \mathrm{H} 7 \mathrm{~B}^{\mathrm{vii}}$ & 2.9178 \\
\hline $\mathrm{F} 3 \mathrm{~B} \cdots \mathrm{C} 16^{\mathrm{i}}$ & $3.365(7)$ & $\mathrm{C} 16 \cdots \mathrm{H} 13$ & 2.5261 \\
\hline $\mathrm{F} 3 \mathrm{~B} \cdots \mathrm{C} 9^{\mathrm{iii}}$ & $3.213(7)$ & $\mathrm{B} 1 \cdots \mathrm{H} 16 \mathrm{~A}^{\mathrm{i}}$ & 2.9217 \\
\hline $\mathrm{F} 4 \mathrm{~B} \cdots \mathrm{C} 15$ & $3.323(8)$ & $\mathrm{B} 1 \cdots \mathrm{H} 1^{\mathrm{ii}}$ & 2.8443 \\
\hline $\mathrm{F} 1 \cdots \mathrm{H} 16 \mathrm{~A}^{\mathrm{i}}$ & 2.7629 & $\mathrm{~B} 1 \cdots \mathrm{H} 2 \mathrm{~B}^{\mathrm{ii}}$ & 2.7082 \\
\hline $\mathrm{F} 1 \cdots \mathrm{H} 2 \mathrm{~B}^{\mathrm{ii}}$ & 2.0078 & $\mathrm{H} 1 \cdots \mathrm{H} 2 \mathrm{~B}$ & 2.2865 \\
\hline $\mathrm{F} 1 \cdots \mathrm{H} 13^{\mathrm{i}}$ & 2.6100 & $\mathrm{H} 1 \cdots \mathrm{F} 2^{\text {viii }}$ & 1.9656 \\
\hline $\mathrm{F} 2 \cdots \mathrm{H} 1^{\mathrm{ii}}$ & 1.9656 & $\mathrm{H} 1 \cdots \mathrm{B} 1^{\mathrm{viii}}$ & 2.8443 \\
\hline F3A $\cdots H 9^{\text {iii }}$ & 2.3818 & $\mathrm{H} 2 \mathrm{~A} \cdots \mathrm{O} 2^{\mathrm{ix}}$ & 2.7789 \\
\hline F3A $\cdots H 14 A^{\mathrm{iii}}$ & 2.8522 & $\mathrm{H} 2 \mathrm{~A} \cdots \mathrm{O} 1^{\mathrm{ix}}$ & 2.1012 \\
\hline $\mathrm{F} 3 \mathrm{~A}^{\cdots} \cdots \mathrm{H} \mathrm{B}^{\mathrm{iii}}$ & 2.8258 & $\mathrm{H} 2 \mathrm{~A} \cdots \mathrm{C} 10^{\mathrm{ix}}$ & 2.7763 \\
\hline $\mathrm{F} 3 \mathrm{~A} \cdots \mathrm{H} 16 \mathrm{~A}^{\mathrm{i}}$ & 2.6715 & $\mathrm{H} 2 \mathrm{~A} \cdots \mathrm{C} 11^{\mathrm{ix}}$ & 3.0749 \\
\hline $\mathrm{F} 3 \mathrm{~A} \cdots \mathrm{H} 16 \mathrm{~B}^{\mathrm{iv}}$ & 2.8039 & $\mathrm{H} 2 \mathrm{~A} \cdots \mathrm{C} 14^{\mathrm{ix}}$ & 2.8183 \\
\hline $\mathrm{F} 3 \mathrm{~B} \cdots \mathrm{H} 16 \mathrm{~B}^{\mathrm{iv}}$ & 2.5723 & $\mathrm{H} 2 \mathrm{~B} \cdots \mathrm{F} 1^{\text {viii }}$ & 2.0078 \\
\hline $\mathrm{F} 3 \mathrm{~B} \cdots \mathrm{H} 2 \mathrm{~B}^{\mathrm{ii}}$ & 2.6183 & $\mathrm{H} 2 \mathrm{~B} \cdots \mathrm{B} 1^{\text {viii }}$ & 2.7082 \\
\hline
\end{tabular}




\begin{tabular}{|c|c|}
\hline $\mathrm{F} 3 \mathrm{~B} \cdots \mathrm{H} \mathrm{B}^{\mathrm{iii}}$ & 2.7261 \\
\hline $\mathrm{F} 3 \mathrm{~B} \cdots \mathrm{H} 9^{\mathrm{iii}}$ & 2.6571 \\
\hline $\mathrm{F} 3 \mathrm{~B} \cdots \mathrm{H} 16 \mathrm{~A}^{\mathrm{i}}$ & 2.8106 \\
\hline $\mathrm{F} 4 \mathrm{~A} \cdots \mathrm{H} 14 \mathrm{~B}^{\mathrm{v}}$ & 2.5018 \\
\hline $\mathrm{F} 4 \mathrm{~B} \cdots \mathrm{H} 16 \mathrm{~A}^{\mathrm{i}}$ & 2.7889 \\
\hline $\mathrm{F} 4 \mathrm{~B} \cdots \mathrm{H} 15 \mathrm{~A}$ & 2.8661 \\
\hline $\mathrm{F} 4 \mathrm{~B} \cdots \mathrm{H} 14 \mathrm{~B}^{\mathrm{v}}$ & 2.6619 \\
\hline $\mathrm{O} 1 \cdots \mathrm{N} 2^{\mathrm{vi}}$ & $2.9345(18)$ \\
\hline $\mathrm{O} 1 \cdots \mathrm{O} 2$ & $2.6600(18)$ \\
\hline $\mathrm{O} 1 \cdots \mathrm{N} 4^{\mathrm{iii}}$ & $2.9210(18)$ \\
\hline $\mathrm{O} 2 \cdots \mathrm{O} 3$ & $2.7108(18)$ \\
\hline $\mathrm{O} 2 \cdots \mathrm{C}^{\mathrm{vii}}$ & $3.171(2)$ \\
\hline $\mathrm{O} 2 \cdots \mathrm{O} 1$ & $2.6600(18)$ \\
\hline $\mathrm{O} 3 \cdots \mathrm{C} 15$ & $3.022(2)$ \\
\hline $\mathrm{O} 3 \cdots \mathrm{O} 2$ & $2.7108(18)$ \\
\hline $\mathrm{O} 1 \cdots \mathrm{H} 4 \mathrm{~B}^{\mathrm{iii}}$ & 2.3604 \\
\hline $\mathrm{O} 1 \cdots \mathrm{H} 2 \mathrm{~A}^{\mathrm{vi}}$ & 2.1012 \\
\hline $\mathrm{O} 2 \cdots \mathrm{H}^{\mathrm{vii}}$ & 2.4983 \\
\hline $\mathrm{O} 2 \cdots \mathrm{H} 2 \mathrm{~A}^{\mathrm{vi}}$ & 2.7789 \\
\hline $\mathrm{O} 3 \cdots \mathrm{H} 15 \mathrm{C}$ & 2.4582 \\
\hline $\mathrm{O} 3 \cdots{ }^{\cdots} 7 \mathrm{~B}^{\mathrm{vii}}$ & 2.7738 \\
\hline $\mathrm{O} 3 \cdots \mathrm{H}^{\mathrm{vii}}$ & 2.6741 \\
\hline $\mathrm{N} 1 \cdots \mathrm{F} 2^{\text {viii }}$ & $2.8445(17)$ \\
\hline $\mathrm{N} 1 \cdots \mathrm{F} 1^{\mathrm{i}}$ & $3.0584(17)$ \\
\hline $\mathrm{N} 2 \cdots \mathrm{F} 1^{\text {viii }}$ & $2.8851(17)$ \\
\hline $\mathrm{N} 2 \cdots \mathrm{O} 1^{\text {ix }}$ & $2.9345(18)$ \\
\hline $\mathrm{N} 3 \cdots \mathrm{F} 1^{\mathrm{i}}$ & $2.9986(17)$ \\
\hline $\mathrm{N} 3{ }^{\cdots} \mathrm{N} 4^{\mathrm{x}}$ & $3.121(2)$ \\
\hline $\mathrm{N} 4 \cdots \mathrm{O} 1^{\mathrm{iii}}$ & $2.9210(18)$ \\
\hline $\mathrm{N} 4 \cdots \mathrm{C} 14^{\mathrm{iii}}$ & $3.385(2)$ \\
\hline $\mathrm{N} 4 \cdots \mathrm{N} 3^{\mathrm{x}}$ & $3.121(2)$ \\
\hline $\mathrm{N} 4 \cdots \mathrm{C} 8$ & $3.418(2)$ \\
\hline $\mathrm{N} 3 \cdots \mathrm{H}_{4} \mathrm{~A}^{\mathrm{x}}$ & 2.2886 \\
\hline $\mathrm{N} 4 \cdots \mathrm{H} 14 \mathrm{~B}^{\mathrm{iii}}$ & 2.9079 \\
\hline $\mathrm{N} 4 \cdots \mathrm{H} 7 \mathrm{~A}$ & 2.7165 \\
\hline $\mathrm{C} 2 \cdots \mathrm{F} 1^{\mathrm{i}}$ & $2.9212(18)$ \\
\hline $\mathrm{C} 4 \cdots \mathrm{F} 1^{\mathrm{i}}$ & $3.136(2)$ \\
\hline $\mathrm{C} 5 \cdots \mathrm{F} 1^{\mathrm{i}}$ & $3.302(2)$ \\
\hline$C 6 \cdots C 6^{x i}$ & $3.594(2)$ \\
\hline $\mathrm{C} 6 \cdots \mathrm{O} 2^{\mathrm{vii}}$ & $3.171(2)$ \\
\hline $\mathrm{C} 6 \cdots \mathrm{F} 1^{\mathrm{i}}$ & $3.239(2)$ \\
\hline $\mathrm{C} 7 \cdots \mathrm{F} 3 \mathrm{~A}^{\mathrm{iii}}$ & $3.282(8)$ \\
\hline $\mathrm{C} 7 \cdots \mathrm{F} 3 \mathrm{~B}^{\mathrm{iii}}$ & $3.279(8)$ \\
\hline $\mathrm{C} 8 \cdots \mathrm{C} 16^{\mathrm{vii}}$ & $3.518(2)$ \\
\hline $\mathrm{C} 8 \cdots \mathrm{N} 4$ & $3.418(2)$ \\
\hline $\mathrm{C} 9 \cdots \mathrm{F} 3 \mathrm{~A}^{\mathrm{iii}}$ & $2.995(9)$ \\
\hline $\mathrm{C} 9 \cdots \mathrm{F} 3 \mathrm{~B}^{\mathrm{iii}}$ & $3.213(7)$ \\
\hline $\mathrm{C} 9 \cdots \mathrm{C} 10^{\mathrm{iii}}$ & $3.552(2)$ \\
\hline
\end{tabular}

$\mathrm{F} 3 \mathrm{~B} \cdots \mathrm{H} 7 \mathrm{~B}^{\mathrm{iii}}$$$
\text { F3B } \cdots \text { H16A }
$$

$\mathrm{F} 4 \mathrm{~A} \cdots \mathrm{H} 14 \mathrm{~B}$

$\mathrm{F} 4 \mathrm{~B} \cdots \mathrm{H} 16 \mathrm{~A}$

$\mathrm{O} 1 \cdots \mathrm{N} 2^{\mathrm{vi}}$

$\mathrm{O} 1 \cdots \mathrm{O} 2$

$\mathrm{O} 1 \cdots \mathrm{N} 4^{\text {iii }}$

$\mathrm{O} 2 \cdots \mathrm{C}^{\text {vi }}$

$\mathrm{O} 2 \cdots \mathrm{O} 1$

$\mathrm{O} 3 \cdots \mathrm{O} 2$

$\mathrm{O} 1 \cdots \mathrm{H}_{4} \mathrm{~B}^{\mathrm{iii}}$

$\mathrm{O} 2 \cdots \mathrm{H} 2 \mathrm{~A}^{\mathrm{vi}}$

$\mathrm{O} 3 \cdots \mathrm{H} 15 \mathrm{C}$

$\mathrm{O} 3 \cdots \mathrm{H}^{7} \mathrm{~B}^{\mathrm{vii}}$

$\mathrm{O} 3 \cdots \mathrm{H} 6^{\text {vii }}$

$\mathrm{N} 2 \cdots \mathrm{F} 1^{\text {viii }}$

$\mathrm{N} 2 \cdots \mathrm{O} 1^{\text {ix }}$

$\mathrm{N} 3 \cdots \mathrm{N}^{\times}$

$\mathrm{N} 4 \cdots \mathrm{O} 1^{\text {ii }}$

$\mathrm{N} 4 \cdots \mathrm{C} 14^{\mathrm{iii}}$

$\mathrm{N} 3 \cdots \mathrm{H}_{4} \mathrm{~A}^{\mathrm{x}}$

$\mathrm{N} 4 \cdots \mathrm{H} 14 \mathrm{~B}^{\mathrm{iii}}$

$\mathrm{N} 4 \cdots \mathrm{H} 7 \mathrm{~A}$

$\mathrm{C} 4 \cdots \mathrm{F} 1$

$\mathrm{C} 5 \cdots \mathrm{F} 1$

C6 ${ }^{\cdots} \mathrm{C}^{\mathrm{xi}}$

$\mathrm{C} 6{ }^{\cdots} \mathrm{O} 2^{\text {vi }}$

$\mathrm{C} 6 \cdots \mathrm{F} 1^{\mathrm{i}}$

C7 $\cdots$ F3 $\mathrm{A}^{\mathrm{iii}}$

$\mathrm{C} 7 \cdots \mathrm{F}^{3} \mathrm{~B}^{\mathrm{ii}}$

$\mathrm{C} 8 \cdots \mathrm{C} 16^{\text {vii }}$

$\mathrm{C} 9 \cdots \mathrm{C} 10$
$\mathrm{H} 2 \mathrm{~B} \cdots \mathrm{H} 1$

H2B $\cdots F^{2} B^{\text {viii }}$

$\mathrm{H} 4 \mathrm{~A} \cdots \mathrm{N}^{\mathrm{x}}$

H4A $\cdots \mathrm{H}^{4} \mathrm{~A}^{\mathrm{x}}$

$\mathrm{H} 4 \mathrm{~B} \cdots \mathrm{C} 7$

$\mathrm{H} 4 \mathrm{~B} \cdots \mathrm{C} 14^{\mathrm{iii}}$

$\mathrm{H} 4 \mathrm{~B} \cdots \mathrm{O} 1^{\mathrm{iii}}$

$\mathrm{H} 4 \mathrm{~B} \cdots \mathrm{C} 8$

H4B $\cdots \mathrm{H} 7 \mathrm{~A}$

$\mathrm{H} 6 \cdots \mathrm{O} 2^{\text {vii }}$

H6 $\cdots$ H7B

$\mathrm{H} 6$ ‥ $3^{\text {vii }}$

H7A $\cdots$ H4B

H7A $\cdots$ N4

H7A $\cdots$ H15 ${ }^{\text {iii }}$

H7A $\cdots \mathrm{H} 9$

H7A $\cdots$ H15B ${ }^{\text {xiii }}$

H7B $\cdots \mathrm{H} 6$

H7B $\cdots 3^{\text {vii }}$

H7B $\cdots \mathrm{C} 16^{\text {vii }}$

H7B $\cdots F 3 A^{\text {iii }}$

H7B $\cdots F^{3} B^{i i i}$

H9 $\cdots$ H14A

H9 $\cdots H 14 C$

H9 $\cdots \mathrm{C} 14$

H9 $\cdots$ H7 A

H9 $\cdots F 3 B^{\text {iii }}$

H9 $\cdots F 3 A^{\text {iii }}$

H9 $\cdots H 15 A^{\text {iii }}$

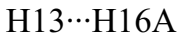

H13 ‥C5

H13 $\cdots \mathrm{C} 6$

$\mathrm{H} 13 \cdots \mathrm{C} 16$

$\mathrm{H} 13 \cdots \mathrm{H} 16 \mathrm{C}$

$\mathrm{H} 13 \cdots \mathrm{F} 1^{\mathrm{i}}$

H14A $\cdots \mathrm{C} 9$

H14A $\cdots$ F3 $A^{\text {iii }}$

H14A $\cdots$ H9

H14B $\cdots$ F4 $\mathrm{B}^{\mathrm{xiv}}$

H14B $\cdots F_{4} A^{\text {xiv }}$

H14B $\cdots N 4^{\text {iii }}$

$\mathrm{H} 14 \mathrm{C} \cdot \mathrm{H} 9$

$\mathrm{H} 14 \mathrm{C} \cdots \mathrm{C} 9$

$\mathrm{H} 14 \mathrm{C} \cdots \mathrm{C} 12^{\mathrm{iii}}$

H14C… C13 $3^{\text {iii }}$

H15A $\cdots$ F4B

H15A $\cdots$ C $^{\text {iii }}$

H15A $\cdots$ H7 $^{\text {iii }}$
2.2865

2.6183

2.2886

2.5725

2.5689

3.0896

2.3604

2.8977

2.2117

2.4983

2.3462

2.6741

2.2117

2.7165

2.4411

2.3813

2.5464

2.3462

2.7738

2.9178

2.8258

2.7261

2.2838

2.2565

2.4843

2.3813

2.6571

2.3818

2.3830

2.3638

2.6875

3.0840

2.5261

2.2633

2.6100

2.7167

2.8522

2.2838

2.6619

2.5018

2.9079

2.2565

2.7394

2.8703

2.9945

2.8661

3.0620

2.4411 
$\mathrm{C} 10 \cdots \mathrm{C} 9^{\text {iii }}$

$\mathrm{C} 12 \cdots \mathrm{C} 13^{\text {vii }}$

$\mathrm{C} 13 \cdots \mathrm{C} 12^{\text {vii }}$

$\mathrm{C} 13 \cdots \mathrm{C} 13^{\text {vii }}$

$\mathrm{C} 14 \cdots \mathrm{N} 4^{\text {iii }}$

$\mathrm{C} 15 \cdots \mathrm{O} 3$

C15 F $4 B$

$\mathrm{C} 16 \cdots \mathrm{F} 3 \mathrm{~B}^{\mathrm{xii}}$

$\mathrm{C} 16 \cdots \mathrm{F} 1^{\mathrm{i}}$

$\mathrm{C} 16 \cdots \mathrm{C} 8^{\text {vii }}$

$\mathrm{C} 16 \cdots \mathrm{F}^{3} \mathrm{~B}^{\mathrm{i}}$

C16 $\cdots$ F3 $A^{\mathrm{i}}$

$\mathrm{C} 5 \cdots \mathrm{H} 13$

C6 $\cdots \mathrm{H} 13$

C7 $\cdots \mathrm{H} 4 \mathrm{~B}$

$\mathrm{C} 8 \cdots \mathrm{H} 4 \mathrm{~B}$

$\mathrm{C} 8 \cdots \mathrm{H} 16 \mathrm{C}^{\mathrm{vii}}$

C9 $\cdots$ H14A

$\mathrm{C} 10-\mathrm{O} 1-\mathrm{C} 14$

$\mathrm{C} 11-\mathrm{O} 2-\mathrm{C} 15$

$\mathrm{C} 12-\mathrm{O} 3-\mathrm{C} 16$

$\mathrm{C} 2-\mathrm{N} 1-\mathrm{C} 6$

$\mathrm{C} 2-\mathrm{N} 3-\mathrm{C} 4$

$\mathrm{C} 2-\mathrm{N} 1-\mathrm{H} 1$

C6- $1-\mathrm{H} 1$

$\mathrm{C} 2-\mathrm{N} 2-\mathrm{H} 2 \mathrm{~A}$

$\mathrm{H} 2 \mathrm{~A}-\mathrm{N} 2-\mathrm{H} 2 \mathrm{~B}$

$\mathrm{C} 2-\mathrm{N} 2-\mathrm{H} 2 \mathrm{~B}$

$\mathrm{H} 4 \mathrm{~A}-\mathrm{N} 4-\mathrm{H} 4 \mathrm{~B}$

$\mathrm{C} 4-\mathrm{N} 4-\mathrm{H} 4 \mathrm{~A}$

$\mathrm{C} 4-\mathrm{N} 4-\mathrm{H} 4 \mathrm{~B}$

$\mathrm{N} 2-\mathrm{C} 2-\mathrm{N} 3$

$\mathrm{N} 1-\mathrm{C} 2-\mathrm{N} 2$

$\mathrm{N} 1-\mathrm{C} 2-\mathrm{N} 3$

$\mathrm{N} 3-\mathrm{C} 4-\mathrm{C} 5$

$\mathrm{N} 4-\mathrm{C} 4-\mathrm{C} 5$

$\mathrm{N} 3-\mathrm{C} 4-\mathrm{N} 4$

$\mathrm{C} 4-\mathrm{C} 5-\mathrm{C} 6$

$\mathrm{C} 6-\mathrm{C} 5-\mathrm{C} 7$

$\mathrm{C} 4-\mathrm{C} 5-\mathrm{C} 7$

$\mathrm{N} 1-\mathrm{C} 6-\mathrm{C} 5$

$\mathrm{C} 5-\mathrm{C} 7-\mathrm{C} 8$

C7-C $8-\mathrm{C} 9$

C7-C $8-\mathrm{C} 13$

$\mathrm{C} 9-\mathrm{C} 8-\mathrm{C} 13$

$\mathrm{C} 8-\mathrm{C} 9-\mathrm{C} 10$

$\mathrm{O} 1-\mathrm{C} 10-\mathrm{C} 9$
$3.552(2)$

3.549 (2)

3.549 (2)

$3.598(2)$

3.385 (2)

$3.022(2)$

$3.323(8)$

$3.318(7)$

3.368 (2)

$3.518(2)$

$3.365(7)$

$3.288(8)$

2.6875

3.0840

2.5689

2.8977

2.8661

2.7167

$116.47(12)$

$114.69(13)$

$116.53(12)$

$120.15(13)$

$118.36(14)$

119.95

119.90

120.02

120.02

119.96

120.02

119.95

120.03

$119.35(15)$

118.59 (14)

$122.06(15)$

$122.06(14)$

$120.69(15)$

$117.24(15)$

$116.06(15)$

$121.62(15)$

$122.30(14)$

$121.27(15)$

$117.06(14)$

$117.45(15)$

$122.04(14)$

120.24 (14)

$119.91(15)$

$123.88(15)$
$\mathrm{H} 15 \mathrm{~A} \cdots \mathrm{H} 9^{\mathrm{iii}}$

$\mathrm{H} 15 \mathrm{~B} \cdots \mathrm{H} 7 \mathrm{~A}^{\mathrm{xv}}$

$\mathrm{H} 15 \mathrm{C} \cdots \mathrm{O} 3$

$\mathrm{H} 15 \mathrm{C} \cdots \mathrm{C} 12$

H16A $\cdots \mathrm{F}^{\mathrm{B}} \mathrm{B}^{\mathrm{i}}$

H16A $\cdots \mathrm{C} 13$

$\mathrm{H} 16 \mathrm{~A} \cdots \mathrm{H} 13$

$\mathrm{H} 16 \mathrm{~A} \cdots \mathrm{F} 1^{\mathrm{i}}$

H16A $\cdots$ F3 $A^{\mathrm{i}}$

$\mathrm{H} 16 \mathrm{~A} \cdots \mathrm{B} 1^{\mathrm{i}}$

H16A $\cdots F 4 B^{i}$

H16B $\cdots F 3 A^{\text {xii }}$

H16B $\cdots F^{3} B^{x i i}$

$\mathrm{H} 16 \mathrm{C} \cdots \mathrm{C} 13$

$\mathrm{H} 16 \mathrm{C} \cdots \mathrm{H} 13$

$\mathrm{H} 16 \mathrm{C} \cdots \mathrm{C}^{\text {vii }}$

H16C $\cdots C 9^{\text {vii }}$

$\mathrm{C} 5-\mathrm{C} 6-\mathrm{H} 6$

$\mathrm{C} 5-\mathrm{C} 7-\mathrm{H} 7 \mathrm{~A}$

$\mathrm{C} 5-\mathrm{C} 7-\mathrm{H} 7 \mathrm{~B}$

$\mathrm{C} 8-\mathrm{C} 7-\mathrm{H} 7 \mathrm{~A}$

$\mathrm{C} 8-\mathrm{C} 7-\mathrm{H} 7 \mathrm{~B}$

$\mathrm{H} 7 \mathrm{~A}-\mathrm{C} 7-\mathrm{H} 7 \mathrm{~B}$

C $8-\mathrm{C} 9-\mathrm{H} 9$

$\mathrm{C} 10-\mathrm{C} 9-\mathrm{H} 9$

$\mathrm{C} 8-\mathrm{C} 13-\mathrm{H} 13$

$\mathrm{C} 12-\mathrm{C} 13-\mathrm{H} 13$

$\mathrm{O} 1-\mathrm{C} 14-\mathrm{H} 14 \mathrm{~A}$

O1-C14-H14B

$\mathrm{O} 1-\mathrm{C} 14-\mathrm{H} 14 \mathrm{C}$

H14A-C14-H14B

$\mathrm{H} 14 \mathrm{~A}-\mathrm{C} 14-\mathrm{H} 14 \mathrm{C}$

$\mathrm{H} 14 \mathrm{~B}-\mathrm{C} 14-\mathrm{H} 14 \mathrm{C}$

$\mathrm{O} 2-\mathrm{C} 15-\mathrm{H} 15 \mathrm{~A}$

$\mathrm{O} 2-\mathrm{C} 15-\mathrm{H} 15 \mathrm{~B}$

$\mathrm{O} 2-\mathrm{C} 15-\mathrm{H} 15 \mathrm{C}$

$\mathrm{H} 15 \mathrm{~A}-\mathrm{C} 15-\mathrm{H} 15 \mathrm{~B}$

$\mathrm{H} 15 \mathrm{~A}-\mathrm{C} 15-\mathrm{H} 15 \mathrm{C}$

$\mathrm{H} 15 \mathrm{~B}-\mathrm{C} 15-\mathrm{H} 15 \mathrm{C}$

$\mathrm{O} 3-\mathrm{C} 16-\mathrm{H} 16 \mathrm{~A}$

O3- $116-\mathrm{H} 16 \mathrm{~B}$

O3- $\mathrm{C} 16-\mathrm{H} 16 \mathrm{C}$

H16A-C16-H16B

$\mathrm{H} 16 \mathrm{~A}-\mathrm{C} 16-\mathrm{H} 16 \mathrm{C}$

$\mathrm{H} 16 \mathrm{~B}-\mathrm{C} 16-\mathrm{H} 16 \mathrm{C}$

$\mathrm{F} 1-\mathrm{B} 1-\mathrm{F} 2$
2.3830

2.5464

2.4582

2.8987

2.8106

2.7409

2.3638

2.7629

2.6715

2.9217

2.7889

2.8039

2.5723

2.7550

2.2633

2.8661

3.0218

119.38

108.05

108.02

108.05

107.99

107.29

120.04

120.05

120.08

120.19

109.47

109.44

109.52

109.44

109.50

109.47

109.52

109.46

109.45

109.47

109.46

109.47

109.46

109.48

109.46

109.47

109.47

109.48

108.92 (14) 


\begin{tabular}{|c|c|c|c|}
\hline $\mathrm{O} 1-\mathrm{C} 10-\mathrm{C} 11$ & $115.49(14)$ & $\mathrm{F} 1-\mathrm{B} 1-\mathrm{F} 3 \mathrm{~A}$ & $111.4(4)$ \\
\hline $\mathrm{C} 9-\mathrm{C} 10-\mathrm{C} 11$ & $120.61(15)$ & $\mathrm{F} 1-\mathrm{B} 1-\mathrm{F} 4 \mathrm{~A}$ & $105.5(4)$ \\
\hline $\mathrm{O} 2-\mathrm{C} 11-\mathrm{C} 12$ & $121.87(15)$ & $\mathrm{F} 1-\mathrm{B} 1-\mathrm{F} 3 \mathrm{~B}$ & $102.4(3)$ \\
\hline $\mathrm{C} 10-\mathrm{C} 11-\mathrm{C} 12$ & $119.09(14)$ & $\mathrm{F} 1-\mathrm{B} 1-\mathrm{F} 4 \mathrm{~B}$ & $116.1(5)$ \\
\hline $\mathrm{O} 2-\mathrm{C} 11-\mathrm{C} 10$ & $118.96(15)$ & $\mathrm{F} 2-\mathrm{B} 1-\mathrm{F} 3 \mathrm{~A}$ & $111.0(3)$ \\
\hline $\mathrm{O} 3-\mathrm{C} 12-\mathrm{C} 11$ & $115.08(14)$ & $\mathrm{F} 2-\mathrm{B} 1-\mathrm{F} 4 \mathrm{~A}$ & $105.9(6)$ \\
\hline $\mathrm{C} 11-\mathrm{C} 12-\mathrm{C} 13$ & $120.37(16)$ & $\mathrm{F} 2-\mathrm{B} 1-\mathrm{F} 3 \mathrm{~B}$ & $104.3(3)$ \\
\hline $\mathrm{O} 3-\mathrm{C} 12-\mathrm{C} 13$ & $124.55(15)$ & $\mathrm{F} 2-\mathrm{B} 1-\mathrm{F} 4 \mathrm{~B}$ & $114.0(3)$ \\
\hline $\mathrm{C} 8-\mathrm{C} 13-\mathrm{C} 12$ & $119.73(15)$ & $\mathrm{F} 3 \mathrm{~A}-\mathrm{B} 1-\mathrm{F} 4 \mathrm{~A}$ & $113.8(7)$ \\
\hline $\mathrm{N} 1-\mathrm{C} 6-\mathrm{H} 6$ & 119.35 & $\mathrm{~F} 3 \mathrm{~B}-\mathrm{B} 1-\mathrm{F} 4 \mathrm{~B}$ & $109.9(4)$ \\
\hline $\mathrm{C} 14-\mathrm{O} 1-\mathrm{C} 10-\mathrm{C} 9$ & $-4.2(2)$ & $\mathrm{C} 6-\mathrm{C} 5-\mathrm{C} 7-\mathrm{C} 8$ & $-103.87(18)$ \\
\hline $\mathrm{C} 14-\mathrm{O} 1-\mathrm{C} 10-\mathrm{C} 11$ & $174.71(14)$ & $\mathrm{C} 5-\mathrm{C} 7-\mathrm{C} 8-\mathrm{C} 13$ & $27.9(2)$ \\
\hline $\mathrm{C} 15-\mathrm{O} 2-\mathrm{C} 11-\mathrm{C} 10$ & $109.92(17)$ & $\mathrm{C} 5-\mathrm{C} 7-\mathrm{C} 8-\mathrm{C} 9$ & $-158.03(14)$ \\
\hline $\mathrm{C} 15-\mathrm{O} 2-\mathrm{C} 11-\mathrm{C} 12$ & $-73.51(19)$ & $\mathrm{C} 13-\mathrm{C} 8-\mathrm{C} 9-\mathrm{C} 10$ & $1.9(2)$ \\
\hline $\mathrm{C} 16-\mathrm{O} 3-\mathrm{C} 12-\mathrm{C} 11$ & $167.55(14)$ & $\mathrm{C} 7-\mathrm{C} 8-\mathrm{C} 13-\mathrm{C} 12$ & $171.44(14)$ \\
\hline $\mathrm{C} 16-\mathrm{O} 3-\mathrm{C} 12-\mathrm{C} 13$ & $-13.3(2)$ & $\mathrm{C} 7-\mathrm{C} 8-\mathrm{C} 9-\mathrm{C} 10$ & $-172.26(14)$ \\
\hline $\mathrm{C} 6-\mathrm{N} 1-\mathrm{C} 2-\mathrm{N} 3$ & $1.2(2)$ & $\mathrm{C} 9-\mathrm{C} 8-\mathrm{C} 13-\mathrm{C} 12$ & $-2.4(2)$ \\
\hline $\mathrm{C} 6-\mathrm{N} 1-\mathrm{C} 2-\mathrm{N} 2$ & $-178.38(15)$ & $\mathrm{C} 8-\mathrm{C} 9-\mathrm{C} 10-\mathrm{C} 11$ & $0.1(2)$ \\
\hline $\mathrm{C} 2-\mathrm{N} 1-\mathrm{C} 6-\mathrm{C} 5$ & $-0.2(2)$ & $\mathrm{C} 8-\mathrm{C} 9-\mathrm{C} 10-\mathrm{O} 1$ & $179.02(14)$ \\
\hline $\mathrm{C} 4-\mathrm{N} 3-\mathrm{C} 2-\mathrm{N} 1$ & $-2.3(2)$ & $\mathrm{C} 9-\mathrm{C} 10-\mathrm{C} 11-\mathrm{O} 2$ & $175.11(14)$ \\
\hline $\mathrm{C} 4-\mathrm{N} 3-\mathrm{C} 2-\mathrm{N} 2$ & $177.29(15)$ & $\mathrm{O} 1-\mathrm{C} 10-\mathrm{C} 11-\mathrm{C} 12$ & $179.46(13)$ \\
\hline $\mathrm{C} 2-\mathrm{N} 3-\mathrm{C} 4-\mathrm{N} 4$ & $-178.46(15)$ & $\mathrm{O} 1-\mathrm{C} 10-\mathrm{C} 11-\mathrm{O} 2$ & $-3.9(2)$ \\
\hline $\mathrm{C} 2-\mathrm{N} 3-\mathrm{C} 4-\mathrm{C} 5$ & $2.5(2)$ & $\mathrm{C} 9-\mathrm{C} 10-\mathrm{C} 11-\mathrm{C} 12$ & $-1.6(2)$ \\
\hline $\mathrm{N} 4-\mathrm{C} 4-\mathrm{C} 5-\mathrm{C} 6$ & $179.43(16)$ & $\mathrm{O} 2-\mathrm{C} 11-\mathrm{C} 12-\mathrm{O} 3$ & $3.7(2)$ \\
\hline $\mathrm{N} 3-\mathrm{C} 4-\mathrm{C} 5-\mathrm{C} 7$ & $176.87(15)$ & $\mathrm{O} 2-\mathrm{C} 11-\mathrm{C} 12-\mathrm{C} 13$ & $-175.57(14)$ \\
\hline $\mathrm{N} 3-\mathrm{C} 4-\mathrm{C} 5-\mathrm{C} 6$ & $-1.5(2)$ & $\mathrm{C} 10-\mathrm{C} 11-\mathrm{C} 12-\mathrm{O} 3$ & $-179.77(14)$ \\
\hline $\mathrm{N} 4-\mathrm{C} 4-\mathrm{C} 5-\mathrm{C} 7$ & $-2.2(2)$ & $\mathrm{C} 10-\mathrm{C} 11-\mathrm{C} 12-\mathrm{C} 13$ & $1.0(2)$ \\
\hline $\mathrm{C} 7-\mathrm{C} 5-\mathrm{C} 6-\mathrm{N} 1$ & $-178.02(15)$ & $\mathrm{O} 3-\mathrm{C} 12-\mathrm{C} 13-\mathrm{C} 8$ & $-178.17(14)$ \\
\hline $\mathrm{C} 4-\mathrm{C} 5-\mathrm{C} 6-\mathrm{N} 1$ & $0.4(2)$ & $\mathrm{C} 11-\mathrm{C} 12-\mathrm{C} 13-\mathrm{C} 8$ & $1.0(2)$ \\
\hline $\mathrm{C} 4-\mathrm{C} 5-\mathrm{C} 7-\mathrm{C} 8$ & $77.84(19)$ & & \\
\hline
\end{tabular}

Symmetry codes: (i) $-x+1,-y+1,-z+1$; (ii) $x, y-1, z+1$; (iii) $-x,-y+1,-z+1$; (iv) $x, y-1, z$; (v) $x+1, y, z$; (vi) $x-1, y, z+1$; (vii) $-x,-y+2,-z+1$; (viii) $x, y+1$, $z^{-1}$; (ix) $x+1, y, z-1$; (x) $-x+1,-y+1,-z$; (xi) $-x,-y+2,-z$; (xii) $x, y+1, z$; (xiii) $x, y, z-1$; (xiv) $x-1, y, z$; (xv) $x, y, z+1$.

Hydrogen-bond geometry $\left(\AA,{ }^{\circ}\right)$

\begin{tabular}{lllll}
\hline$D-\mathrm{H} \cdots A$ & $D-\mathrm{H}$ & $\mathrm{H} \cdots A$ & $D \cdots A$ & $D-\mathrm{H}^{\cdots} A$ \\
\hline $\mathrm{N} 1-\mathrm{H} 1 \cdots \mathrm{F} 2^{\text {viii }}$ & 0.88 & 1.97 & $2.8445(17)$ & 177 \\
$\mathrm{~N} 2-\mathrm{H} 2 A \cdots \mathrm{O} 1^{\text {ix }}$ & 0.88 & 2.10 & $2.9345(18)$ & 158 \\
$\mathrm{~N} 2-\mathrm{H} 2 B \cdots \mathrm{F} 1^{\text {viii }}$ & 0.88 & 2.01 & $2.8851(17)$ & 174 \\
$\mathrm{~N} 4-\mathrm{H} 4 A \cdots \mathrm{N} 3^{\mathrm{x}}$ & 0.88 & 2.29 & $3.121(2)$ & 158 \\
$\mathrm{~N} 4-\mathrm{H} 4 B \cdots \mathrm{O} 1^{\text {iii }}$ & 0.88 & 2.36 & $2.9210(18)$ & 122 \\
$\mathrm{C} 6-\mathrm{H} 6 \cdots \mathrm{O} 2^{\text {vii }}$ & 0.95 & 2.50 & $3.171(2)$ & 128 \\
$\mathrm{C} 9-\mathrm{H} 9 \cdots \mathrm{F} 3 A^{\text {iii }}$ & 0.95 & 2.38 & $2.995(9)$ & 122 \\
$\mathrm{C} 14-\mathrm{H} 14 B \cdots \mathrm{F} 4 A^{\mathrm{xiv}}$ & 0.98 & 2.50 & $3.414(10)$ & 155 \\
$\mathrm{C} 15-\mathrm{H} 15 C \cdots \mathrm{O} 3$ & 0.98 & 2.46 & $3.022(2)$ & 116 \\
\hline
\end{tabular}

Symmetry codes: (iii) $-x,-y+1,-z+1$; (vii) $-x,-y+2,-z+1$; (viii) $x, y+1, z-1$; (ix) $x+1, y, z-1$; (x) $-x+1,-y+1,-z$; (xiv) $x-1, y, z$. 